\title{
Servicios otorgados en unidades de la Secretaría de Salud, 2000
}

M ediante el Sistema de Información en Salud para Población Abierta (SISPA), la Secretaría de Salud (SSA) obtiene estadísticas sobre las actividades que desarrolla, así como de los recursos humanos, físicos y materiales de que dispone. De esta manera el SISPA establece lo que debe registrarse, cuándo y en qué forma debe hacerse, cómo y cuándo se deben procesar los datos para convertirlos en estadística, la periodicidad y el flujo de los reportes.

Al SISPA lo integran los siguientes subsistemas: población, daños a la salud, prestación de servicios y recursos disponibles en la SSA, los cuales responden a las necesidades de información en salud; su propósito es generar e integrar información homogénea, relevante, oportuna y confiable, útil para proporcionar apoyo a tomadores de decisiones e investigadores en salud.

El Subsistema de Prestación de Servicios cuenta con una estructura piramidal, ya que proporciona información con diferente desagregación a los distintos niveles administrati- vos, y se refiere a las atenciones que se brindan en la Secretaría de Salud a la población demandante.

Conforman este subsistema los formatos primarios, intermedios y reportes de actividades, que son actualizados de acuerdo con los requerimientos de los usuarios. El flujo de información se realiza con la siguiente periodicidad: el corte en la unidad aplicativa se hace el 25 de cada mes (mes estadístico), de tal modo que la información debe tenerse en el estado el día 30, para que este último la envíe al nivel nacional el 15 del mes siguiente, y en la Dirección General de Información y Evaluación del Desempeño se cierre el día 30.

En el estado se concentran las actividades que rinde cada unidad aplicativa de la SSA al nivel inmediato superior, por lo que en el nivel nacional se recibe mensualmente un informe de actividades por entidad federativa y nivel de atención, lo que permite conocer la cobertura e impacto del servicio, así como la evaluación de la operación.
A continuación se presenta una muestra de la información correspondiente al Subsistema de Prestación de Servicios del año 2000. La primera parte contiene las atenciones otorgadas como consulta externa, hospitalización y servicios auxiliares de diagnóstico; la otra, los tabulados con diferentes poblaciones de acuerdo con los servicios de los que han hecho uso, y se refiere a los menores de cinco años, la atención materna, los servicios de planificación familiar, los accidentes y las detecciones de las distintas enfermedades.

Cabe mencionar que este subsistema capta una gran cantidad de información y se encuentra disponible en el sitio de la SSA en internet, así como en las tabulaciones que se generan mensualmente para cada programa sustantivo de la SSA y en las publicaciones de la Dirección General de Información y Evaluación del Desempeño, en Leibnitz 20, 3er. Piso, colonia Nueva Anzures, 11590 México, D.F. 


\section{Consulta externa otorgada. México, 2000}

\begin{tabular}{|c|c|c|c|c|c|c|c|c|}
\hline & & & Enfermedades & & Consulta & Planificación & Salud & Salud \\
\hline Entidad federativa & Total & Transmisibles & Crónico degenerativas & Otras & a sanos & familiar* & bucal & mental \\
\hline Estados Unidos Mexicanos & 70297462 & 14898532 & 3663546 & 22013240 & 18609001 & 5727252 & 4664280 & 726864 \\
\hline Aguascalientes & 530958 & 122474 & 46195 & 147982 & 116036 & 37980 & 45823 & 14468 \\
\hline Baja California & 739096 & 160167 & 56545 & 223663 & 153689 & 79322 & 59258 & 6452 \\
\hline Baja California Sur & 344060 & 66127 & 26286 & 97942 & 94342 & 34992 & 18794 & 5577 \\
\hline Campeche & 734312 & 177855 & 32962 & 240837 & 175337 & 54154 & 44692 & 8475 \\
\hline Coahuila & 1013444 & 257669 & 67538 & 316307 & 163346 & 118154 & 81991 & 8439 \\
\hline Colima & 455645 & 109926 & 31772 & 153089 & 89850 & 37567 & 30631 & 2810 \\
\hline Chiapas & 2143178 & 487150 & 68904 & 702953 & 531015 & 181636 & 160395 & 11125 \\
\hline Chihuahua & 1343785 & 238904 & 98379 & 445574 & 349029 & 128443 & 67476 & 15980 \\
\hline Distrito Federal & 6300445 & 851171 & 210323 & 3067437 & 938331 & 228726 & 725796 & 278661 \\
\hline Durango & 842385 & 208871 & 67201 & 248432 & 161893 & 95192 & 50228 & 10568 \\
\hline Guanajuato & 2953804 & 803291 & 147190 & 823983 & 843446 & 186506 & 133541 & 15847 \\
\hline Guerrero & 3748386 & 836710 & 143128 & 1035546 & 1203986 & 398688 & 121615 & 8713 \\
\hline Hidalgo & 2545440 & 631808 & 108611 & 624408 & 786402 & 155450 & 225957 & 12804 \\
\hline Jalisco & 4060611 & 782235 & 333983 & 1207830 & 1182417 & 323814 & 187618 & 42714 \\
\hline México & 9434066 & 1936114 & 271837 & 1834700 & 3460571 & 1406421 & 502445 & 21978 \\
\hline Michoacán & 3103807 & 757564 & 212698 & 1000489 & 686725 & 189799 & 242917 & 13615 \\
\hline Morelos & 1199898 & 341774 & 50638 & 389250 & 226959 & 80314 & 105854 & 5109 \\
\hline $\bar{N}$ ayarit & 961186 & 169881 & 73392 & 245803 & 335765 & 90706 & 42310 & 3329 \\
\hline N uevo León & 2151910 & 429060 & 173050 & 615443 & 591076 & 155404 & 158708 & 29169 \\
\hline 0 axaca & 2538638 & 650350 & 117252 & 803282 & 624568 & 179382 & 145952 & 17852 \\
\hline Puebla & 2962498 & 679866 & 150849 & 737583 & 879346 & 193906 & 306206 & 14742 \\
\hline Q uerétaro & 1224797 & 293173 & 53590 & 369206 & 384694 & 53467 & 54798 & 15869 \\
\hline Q uintana Roo & 694550 & 157147 & 30025 & 194239 & 192212 & 76248 & 44040 & 639 \\
\hline San Luis Potosí & 1332405 & 230325 & 117635 & 325094 & 418180 & 97746 & 136723 & 6702 \\
\hline Sinaloa & 1344403 & 283425 & 95056 & 414906 & 355425 & 94463 & 82702 & 18426 \\
\hline Sonora & 1323122 & 320659 & 92828 & 461220 & 248711 & 88482 & 77285 & 33937 \\
\hline Tabasco & 3014499 & 555495 & 120465 & 923723 & 927232 & 202885 & 261115 & 23584 \\
\hline Tamaulipas & 1634106 & 354669 & 151140 & 586792 & 353417 & 105930 & 65637 & 16521 \\
\hline Tlaxcala & 1269831 & 346323 & 58243 & 338449 & 368322 & 82248 & 69594 & 6652 \\
\hline Veracruz & 4818607 & 1095755 & 298325 & 1656237 & 1143244 & 374448 & 232728 & 17870 \\
\hline Yucatán & 1298212 & 327991 & 69258 & 375443 & 355261 & 88549 & 48676 & 33034 \\
\hline Zacatecas & 1091517 & 234603 & 88248 & 329635 & 268174 & 100977 & 64677 & 5203 \\
\hline Institutos nacionales & 1143861 & 0 & 0 & 1075763 & 0 & 5253 & 68098 & 0 \\
\hline
\end{tabular}

* Se refiere a consultas cuyo único motivo fue planificación familiar. Difiere del presentado en el cuadro VI, que contiene atenciones colaterales y lo correspondiente a Estrategia de Extensión de Cobertura

Fuente: Secretaría de Salud (SSA), C oordinación de Planeación Estratégica, D irección General de Información y Evaluación del D esempeño (D G IE), Sistema de Información en Salud para Población Abierta (SISPA) 


\section{Servicios de hospitalización.* México, 2000}

\begin{tabular}{|c|c|c|c|c|c|c|c|c|}
\hline & & & & Intervenciones & Porcentaje de & Promedio de & \multicolumn{2}{|c|}{ M ortalidad hospitalaria } \\
\hline Entidad federativa & Egresos & Días paciente & Días estancia & quirúrgicas & ocupacion & días estancia & Total & Ajustada \\
\hline Estados Unidos Mexicanos & 1455013 & 6700089 & 6011776 & 849326 & 71.24 & 4.13 & 26.07 & 14.07 \\
\hline Aguascalientes & 27409 & 106924 & 84441 & 11975 & 75.00 & 3.08 & 17.59 & 12.33 \\
\hline Baja California & 26545 & 110825 & 93596 & 15008 & 97.21 & 3.53 & 42.04 & 27.16 \\
\hline Baja California Sur & 8915 & 37285 & 34537 & 5438 & 56.19 & 3.87 & 25.57 & 16.15 \\
\hline Campeche & 17731 & 57935 & 51627 & 14394 & 65.96 & 2.91 & 22.39 & 11.45 \\
\hline Coahuila & 22280 & 82392 & 76878 & 10303 & 59.48 & 3.45 & 15.93 & 7.45 \\
\hline Colima & 13320 & 42516 & 39116 & 8225 & 59.35 & 2.94 & 23.95 & 12.09 \\
\hline Chiapas & 51796 & 189755 & 152136 & 30216 & 88.72 & 2.94 & 28.92 & 12.82 \\
\hline Chihuahua & 45016 & 195244 & 183448 & 22211 & 76.69 & 4.08 & 33.92 & 15.28 \\
\hline Distrito Federal & 195177 & 1080535 & 1056208 & 114817 & 66.50 & 5.41 & 29.19 & 13.25 \\
\hline Durango & 25052 & 118646 & 97808 & 13003 & 67.26 & 3.90 & 26.86 & 14.65 \\
\hline Guanajuato & 62469 & 221515 & 274134 & 58832 & 74.81 & 4.39 & 20.25 & 12.34 \\
\hline Guerrero & 43007 & 157320 & 130270 & 24678 & 70.77 & 3.03 & 29.55 & 14.21 \\
\hline Hidalgo & 28899 & 207047 & 186595 & 14647 & 85.04 & 6.46 & 28.31 & 14.91 \\
\hline Jalisco & 64272 & 259149 & 197454 & 45056 & 74.29 & 3.07 & 14.69 & 9.37 \\
\hline México & 123745 & 780015 & 657964 & 77804 & 59.18 & 5.32 & 27.98 & 18.93 \\
\hline Michoacán & 57942 & 214611 & 192417 & 29561 & 70.50 & 3.32 & 24.73 & 15.29 \\
\hline Morelos & 26460 & 107832 & 79310 & 14866 & 88.10 & 3.00 & 29.44 & 15.91 \\
\hline $\mathrm{N}$ ayarit & 13674 & 41474 & 36990 & 8219 & 55.65 & 2.71 & 33.06 & 20.40 \\
\hline Nuevo León & 38077 & 153451 & 131148 & 19721 & 71.42 & 3.44 & 16.05 & 10.19 \\
\hline 0 axaca & 36072 & 150766 & 143921 & 21400 & 77.19 & 3.99 & 21.01 & 13.61 \\
\hline Puebla & 33432 & 231190 & 151914 & 18568 & 70.75 & 4.54 & 28.33 & 18.34 \\
\hline Q uerétaro & 25783 & 104668 & 79415 & 19132 & 84.52 & 3.08 & 28.31 & 18.69 \\
\hline Q uintana Roo & 14884 & 50754 & 43410 & 8011 & 69.76 & 2.92 & 21.03 & 10.28 \\
\hline San Luis Potosí & 24966 & 69135 & 69977 & 10995 & 78.09 & 2.80 & 18.51 & 8.49 \\
\hline Sinaloa & 33149 & 132077 & 110285 & 22950 & 77.40 & 3.33 & 29.11 & 19.31 \\
\hline Sonora & 56710 & 240923 & 202784 & 21103 & 66.00 & 3.58 & 18.16 & 11.69 \\
\hline Tabasco & 55075 & 231314 & 214665 & 32961 & 86.63 & 3.90 & 26.35 & 16.47 \\
\hline Tamaulipas & 66536 & 241005 & 217375 & 35429 & 65.09 & 3.27 & 26.30 & 15.00 \\
\hline Tlaxcala & 22102 & 56477 & 57139 & 11483 & 72.30 & 2.59 & 20.59 & 12.35 \\
\hline Veracruz & 95097 & 387806 & 344569 & 56415 & 72.73 & 3.62 & 30.45 & 16.77 \\
\hline Yucatán & 20639 & 131756 & 124024 & 12709 & 75.00 & 6.01 & 23.26 & 17.25 \\
\hline Zacatecas & 19479 & 61938 & 46493 & 9394 & 95.58 & 2.39 & 19.25 & 10.58 \\
\hline Institutos nacionales & 59303 & 445809 & 449728 & 29802 & 83.00 & 7.58 & 33.57 & ND \\
\hline
\end{tabular}

Fuente: Secretaría de Salud (SSA), C o ordinación de Planeación Estratégica, D irección General de Información y Evaluación del Desempeño (DG IE), Sistema de Información en Salud para Población A bierta (SISPA) 


\section{Servicios auxiliares de diagnóstico. México, 2000}

\begin{tabular}{|c|c|c|c|c|c|c|c|c|c|c|}
\hline \multirow[b]{2}{*}{ Entidad federativa } & \multicolumn{2}{|c|}{ Análisis clínicos } & \multicolumn{2}{|c|}{ Rayos X } & \multicolumn{2}{|c|}{ Anatomía patológica } & \multicolumn{2}{|c|}{ Electrocardiograma } & \multicolumn{2}{|c|}{ Ultrasonido } \\
\hline & Estudios & Personas & Estudios & Personas & Estudios & Personas & Estudios & Personas & Estudios & Personas \\
\hline tados Unidos Mexicanos & 41201441 & 12965620 & 3078661 & 2329942 & 654200 & 427884 & 185789 & 180218 & 365602 & 356065 \\
\hline
\end{tabular}

\begin{tabular}{|c|c|c|c|c|c|c|c|c|c|c|}
\hline A guascalientes & 713192 & 253279 & 57651 & 46648 & 3462 & 3429 & 5619 & 5339 & 6765 & 6753 \\
\hline Baja California & 537090 & 154896 & 48794 & 34586 & 7645 & 6060 & 8939 & 7306 & 3936 & 3893 \\
\hline Baja California Sur & 266402 & 154256 & 26468 & 22091 & 8442 & 8442 & 1895 & 1834 & 4240 & 4240 \\
\hline Campeche & 440576 & 163230 & 37493 & 26863 & 3538 & 2324 & 1362 & 1362 & 7967 & 7788 \\
\hline Coahuila & 610852 & 210878 & 29886 & 24662 & 37079 & 13997 & 2217 & 2134 & 8889 & 8883 \\
\hline Colima & 208037 & 108584 & 21901 & 18895 & 1884 & 1884 & 2620 & 2412 & 3287 & 3271 \\
\hline Chiapas & 941573 & 296490 & 63769 & 44591 & 21764 & 4471 & 3758 & 3705 & 3896 & 3847 \\
\hline Chihuahua & 1094419 & 287453 & 103967 & 82256 & 7314 & 7128 & 8519 & 7855 & 11496 & 11306 \\
\hline Distrito Federal & 8018001 & 2413531 & 638038 & 526061 & 208511 & 98469 & 33398 & 32790 & 78259 & 75435 \\
\hline Durango & 502823 & 209692 & 54146 & 46482 & 5111 & 3883 & 5057 & 4766 & 9281 & 8967 \\
\hline Guanajuato & 1034322 & 377131 & 113878 & 86990 & 45783 & 45773 & 2896 & 2894 & 12139 & 12129 \\
\hline Guerrero & 1359813 & 572728 & 75115 & 48837 & 2579 & 2558 & 3010 & 2962 & 8011 & 7980 \\
\hline Hidalgo & 678771 & 313705 & 48238 & 38501 & 3094 & 3094 & 772 & 772 & 12860 & 12153 \\
\hline Jalisco & 1126881 & 462676 & 87248 & 65459 & 40905 & 35315 & 6699 & 6517 & 9861 & 9850 \\
\hline México & 2686008 & 1009078 & 202015 & 152358 & 27372 & 26776 & 14461 & 14253 & 20920 & 19672 \\
\hline Michoacán & 1062739 & 542302 & 115809 & 86191 & 7429 & 7429 & 8790 & 8652 & 16085 & 15717 \\
\hline Morelos & 568140 & 173651 & 62189 & 48683 & 4724 & 4723 & 5307 & 5307 & 5056 & 5056 \\
\hline $\mathrm{N}$ ayarit & 344667 & 144537 & 38121 & 25631 & 6314 & 2116 & 2108 & 1936 & 2870 & 2732 \\
\hline Nuevo León & 1041004 & 381165 & 106651 & 82780 & 7843 & 7843 & 7171 & 7001 & 3835 & 3828 \\
\hline 0 axaca & 597877 & 194261 & 66473 & 47839 & 9618 & 4236 & 3627 & 3605 & 8236 & 8224 \\
\hline Puebla & 990016 & 405783 & 67570 & 48884 & 15744 & 7731 & 3930 & 3925 & 10394 & 10215 \\
\hline Q uerétaro & 585072 & 185680 & 55611 & 47833 & 7092 & 4150 & 2587 & 2587 & 8940 & 8818 \\
\hline Q uintana Roo & 447377 & 263772 & 28538 & 24536 & 1554 & 1554 & 962 & 962 & 6933 & 6932 \\
\hline San Luis Potosí & 608489 & 255501 & 30551 & 26894 & 1440 & 1439 & 2776 & 2742 & 3211 & 3211 \\
\hline Sinaloa & 846879 & 255174 & 72149 & 56744 & 4087 & 3926 & 4451 & 4399 & 12809 & 12756 \\
\hline Sonora & 1202256 & 369921 & 98494 & 73597 & 7245 & 7242 & 4570 & 4543 & 3468 & 3388 \\
\hline Tabasco & 1414250 & 445519 & 92014 & 72358 & 11151 & 10978 & 5551 & 5551 & 15788 & 15721 \\
\hline Tamaulipas & 1297050 & 554883 & 109769 & 88441 & 34246 & 33953 & 13318 & 12711 & 17313 & 17007 \\
\hline Tlaxcala & 496479 & 178467 & 38797 & 25277 & 5719 & 3393 & 1771 & 1771 & 7535 & 6861 \\
\hline Veracruz & 2570151 & 703734 & 144622 & 102891 & 28833 & 27339 & 10939 & 10930 & 25870 & 24076 \\
\hline Yucatán & 641274 & 260532 & 36621 & 31833 & 4595 & 4595 & 2574 & 2574 & 9164 & 9107 \\
\hline Zacatecas & 514051 & 174764 & 45973 & 38847 & 5722 & 5366 & 4135 & 4121 & 6288 & 6249 \\
\hline Institutos nacionales & 5754910 & 488367 & 260102 & 135403 & 66361 & 26268 & 0 & 0 & 0 & 0 \\
\hline
\end{tabular}

Fuente: Secretaría de Salud (SSA), C oordinación de Planeación Estratégica, D irección General de Información y Evaluación del Desempeño (D GIE), Sistema de Información en Salud para Población A bierta (SISPA) 


\section{Atención a menores de Cinco años. México, 2000}

\begin{tabular}{|c|c|c|c|c|c|c|c|c|}
\hline \multirow[b]{2}{*}{ Entidad federativa } & \multirow{2}{*}{$\begin{array}{l}\text { Consulta a } \\
\text { niño sano }\end{array}$} & \multicolumn{3}{|c|}{$\begin{array}{l}\text { Consulta según } \\
\text { presencia de desnutrición }\end{array}$} & \multicolumn{2}{|c|}{$\begin{array}{l}\text { Consultas enfermedad } \\
\text { diarreica aguda* }\end{array}$} & \multicolumn{2}{|c|}{$\begin{array}{l}\text { Consultas Infección } \\
\text { respiratoria aguda* }\end{array}$} \\
\hline & & Total & Sin desnutrición & Con desnutrición & Total & Primera vez & Total & Primera vez \\
\hline Estados Unidos Mexicanos & 5473660 & 14246641 & 11458489 & 2788152 & 1028649 & 884803 & 5091570 & 4209759 \\
\hline
\end{tabular}

\begin{tabular}{|c|c|c|c|c|c|c|c|c|}
\hline Aguascalientes & 23062 & 87918 & 79625 & 8293 & 8081 & 7186 & 44052 & 37764 \\
\hline Baja California & 31684 & 123886 & 118801 & 5085 & 11362 & 9946 & 62563 & 52093 \\
\hline Baja California Sur & 17408 & 50268 & 47443 & 2825 & 5032 & 4207 & 21742 & 16681 \\
\hline Campeche & 44733 & 159706 & 116362 & 43344 & 11500 & 10281 & 66306 & 55153 \\
\hline Coahuila & 35779 & 146247 & 133169 & 13078 & 13912 & 11599 & 90823 & 76189 \\
\hline Colima & 22413 & 82444 & 74958 & 7486 & 4686 & 3946 & 34423 & 26814 \\
\hline Chiapas & 180000 & 446479 & 303746 & 142733 & 69549 & 63821 & 153604 & 136650 \\
\hline Chihuahua & 107458 & 269463 & 227860 & 41603 & 24580 & 20712 & 98571 & 84421 \\
\hline Distrito Federal & 149956 & 532966 & 505760 & 27206 & 63991 & 57739 & 374114 & 328651 \\
\hline Durango & 31355 & 135057 & 125712 & 9345 & 16794 & 14800 & 76359 & 64256 \\
\hline Guanajuato & 233117 & 780864 & 647495 & 133369 & 53860 & 47479 & 346925 & 292929 \\
\hline Guerrero & 423809 & 1047552 & 619132 & 428420 & 72379 & 60186 & 234602 & 186587 \\
\hline Hidalgo & 214127 & 499285 & 386243 & 113042 & 21902 & 18969 & 201530 & 164565 \\
\hline Jalisco & 290808 & 776954 & 684914 & 92040 & 42286 & 37134 & 297957 & 252325 \\
\hline México & 1190618 & 2485284 & 2122302 & 362982 & 160787 & 135371 & 729399 & 589104 \\
\hline Michoacán & 182307 & 575494 & 480235 & 95259 & 47867 & 42416 & 225246 & 194168 \\
\hline Morelos & 62481 & 239218 & 205415 & 33803 & 17203 & 15464 & 108994 & 94458 \\
\hline $\mathrm{N}$ ayarit & 91740 & 187425 & 159638 & 27787 & 14465 & 11916 & 49835 & 39762 \\
\hline Nuevo León & 150670 & 384030 & 366291 & 17739 & 14319 & 12772 & 160645 & 140022 \\
\hline 0 axaca & 182004 & 548217 & 371668 & 176549 & 56766 & 49181 & 177007 & 146205 \\
\hline Puebla & 284940 & 717903 & 543552 & 174351 & 45909 & 38005 & 226055 & 172972 \\
\hline Q uerétaro & 131283 & 339304 & 242139 & 97165 & 18265 & 15974 & 108901 & 90514 \\
\hline Q uintana Roo & 55940 & 142268 & 97979 & 44289 & 7077 & 6213 & 52073 & 43199 \\
\hline San Luis Potosí & 167563 & 349827 & 250195 & 99632 & 17750 & 15690 & 87975 & 72660 \\
\hline Sinaloa & 89116 & 261585 & 234162 & 27423 & 21677 & 18836 & 92743 & 76463 \\
\hline Sonora & 54037 & 269688 & 257790 & 11898 & 28124 & 24118 & 125260 & 103174 \\
\hline Tabasco & 287147 & 640507 & 513689 & 126818 & 29897 & 24578 & 150539 & 116505 \\
\hline Tamaulipas & 79738 & 268470 & 246322 & 22148 & 23322 & 20138 & 122932 & 105186 \\
\hline Tlaxcala & 105776 & 294407 & 253599 & 40808 & 14850 & 11214 & 115262 & 84541 \\
\hline Veracruz & 337844 & 984110 & 770801 & 213309 & 50899 & 41046 & 269664 & 212238 \\
\hline Yucatán & 142789 & 308473 & 190185 & 118288 & 19132 & 16352 & 102074 & 83983 \\
\hline Zacatecas & 71958 & 111342 & 81307 & 30035 & 19105 & 16193 & 83395 & 69527 \\
\hline Institutos nacionales & 0 & 0 & 0 & 0 & 1321 & 1321 & 0 & 0 \\
\hline
\end{tabular}

* Incluye Estrategia de Extensión de Cobertura

Fuente: Secretaría de Salud (SSA), Coordinación de Planeación Estratégica, Dirección General de Información y Evaluación del Desempeño (DGIE), Sistema de Información en Salud para Población A bierta (SISPA) 


\section{Atencion materna. México, 2000}

\begin{tabular}{|c|c|c|c|c|c|c|c|c|}
\hline \multirow[b]{2}{*}{ Entidad federativa } & \multirow{2}{*}{$\begin{array}{c}\text { Embarazadas } \\
\text { atendidas* }\end{array}$} & \multirow{2}{*}{$\begin{array}{l}\text { Consultas a } \\
\text { embarazadas* }\end{array}$} & \multicolumn{3}{|c|}{ Nacimientos atendidos } & \multicolumn{2}{|c|}{ Nacidos vivos } & \multirow{2}{*}{$\begin{array}{l}\text { Abortos } \\
\text { atendidos* }\end{array}$} \\
\hline & & & Total* & Parto* & Cesárea & Total* & Con bajo peso & \\
\hline Estados Unidos Mexicanos & 1216649 & 4224393 & 614995 & 457277 & 157718 & 610647 & 45611 & 71641 \\
\hline Aguascalientes & 13207 & 45783 & 10295 & 6872 & 3423 & 10270 & 937 & 1261 \\
\hline Baja California & 16456 & 49734 & 12895 & 10339 & 2556 & 12779 & 793 & 1346 \\
\hline Baja California Sur & 8261 & 28099 & 4174 & 2983 & 1191 & 4179 & 222 & 541 \\
\hline Campeche & 9979 & 49904 & 6582 & 4748 & 1834 & 6551 & 374 & 714 \\
\hline Coahuila & 17217 & 56189 & 9926 & 7299 & 2627 & 9896 & 592 & 1382 \\
\hline Colima & 8439 & 28701 & 5350 & 3799 & 1551 & 5320 & 310 & 648 \\
\hline Chiapas & 40219 & 144372 & 23896 & 17145 & 6751 & 23632 & 1805 & 3112 \\
\hline Chihuahua & 22823 & 68679 & 11291 & 7866 & 3425 & 11266 & 1029 & 1137 \\
\hline Distrito Federal & 77978 & 208569 & 62518 & 44800 & 17718 & 62071 & 5255 & 9370 \\
\hline Durango & 15958 & 54489 & 9505 & 7170 & 2335 & 9466 & 654 & 1383 \\
\hline Guanajuato & 70182 & 245547 & 34801 & 26745 & 8056 & 34601 & 2697 & 4025 \\
\hline Guerrero & 56824 & 193188 & 24842 & 20459 & 4383 & 24685 & 1899 & 2362 \\
\hline Hidalgo & 34655 & 126212 & 13792 & 10354 & 3438 & 13690 & 1244 & 1586 \\
\hline Jalisco & 78035 & 335343 & 34489 & 26879 & 7610 & 34443 & 1692 & 3739 \\
\hline México & 191586 & 524502 & 62330 & 49290 & 13040 & 61699 & 4851 & 7580 \\
\hline Michoacán & 57680 & 178995 & 26586 & 20712 & 5874 & 26383 & 1763 & 3043 \\
\hline Morelos & 15218 & 57889 & 10643 & 7648 & 2995 & 10503 & 909 & 1480 \\
\hline $\mathrm{N}$ ayarit & 15941 & 61427 & 7544 & 6048 & 1496 & 7550 & 464 & 850 \\
\hline Nuevo León & 32661 & 141586 & 16905 & 10744 & 6161 & 16748 & 1103 & 1721 \\
\hline 0 axaca & 45233 & 157401 & 17580 & 12485 & 5095 & 17351 & 1289 & 1805 \\
\hline Puebla & 52355 & 187488 & 21324 & 17184 & 4140 & 21068 & 1682 & 2258 \\
\hline Querétaro & 23233 & 79996 & 13352 & 9508 & 3844 & 13276 & 1240 & 1494 \\
\hline Q uintana Roo & 15811 & 48806 & 7240 & 5332 & 1908 & 7213 & 543 & 1015 \\
\hline San Luis Potosí & 22806 & 92875 & 13059 & 10141 & 2918 & 12999 & 982 & 1477 \\
\hline Sinaloa & 25802 & 79524 & 11656 & 8377 & 3279 & 11625 & 762 & 1559 \\
\hline Sonora & 27419 & 85376 & 19136 & 14426 & 4710 & 19026 & 987 & 1936 \\
\hline Tabasco & 45854 & 182642 & 20838 & 14339 & 6499 & 20633 & 1503 & 2289 \\
\hline Tamaulipas & 34868 & 115703 & 24375 & 17182 & 7193 & 24274 & 1512 & 3047 \\
\hline Tlaxcala & 19283 & 64936 & 10421 & 7794 & 2627 & 10318 & 912 & 1328 \\
\hline Veracruz & 77518 & 353133 & 42168 & 31299 & 10869 & 41727 & 2777 & 3876 \\
\hline Yucatán & 20516 & 78224 & 9849 & 6560 & 3289 & 9752 & 1026 & 543 \\
\hline Zacatecas & 18078 & 71156 & 10681 & 8734 & 1947 & 10644 & 767 & 1318 \\
\hline Institutos nacionales & 4554 & 27925 & 4952 & 2016 & 2936 & 5009 & 1036 & 416 \\
\hline
\end{tabular}

* Incluye Estrategia de Extensión de Cobertura

Fuente: Secretaría de Salud (SSA), C oordinación de Planeación Estratégica, D irección General de Información y Evaluación del D esempeño (D G IE), Sistema de Información en Salud para Población A bierta (SISPA) 


\section{Servicios de planificación familiar. México, 2000}

\begin{tabular}{|c|c|c|c|c|c|c|c|}
\hline \multirow[b]{2}{*}{ Entidad federativa } & \multirow{2}{*}{$\begin{array}{l}\text { Consultas y } \\
\text { atenciones* }\end{array}$} & \multirow{2}{*}{$\begin{array}{c}\text { Nuevos } \\
\text { aceptantes* }\end{array}$} & \multirow{2}{*}{$\begin{array}{l}\text { Usuarias } \\
\text { activas* }\end{array}$} & \multicolumn{3}{|c|}{ Intervenciones quirúrgicas } & \multirow{2}{*}{$\begin{array}{l}\text { Aceptantes post- } \\
\text { evento obstétrico }\end{array}$} \\
\hline & & & & Total & Oclusión tubaria & Vasectomía & \\
\hline Estados Unidos Mexicanos & 7994806 & 904941 & 2762345 & 105883 & 100762 & 5121 & 277493 \\
\hline Aguascalientes & 95295 & 8336 & 22775 & 1171 & 1073 & 98 & 2751 \\
\hline Baja California & 93806 & 22916 & 33210 & 1891 & 1711 & 180 & 6667 \\
\hline Baja C alifornia Sur & 41987 & 5609 & 16204 & 771 & 723 & 48 & 1503 \\
\hline Campeche & 77777 & 6327 & 26025 & 2093 & 2025 & 68 & 3918 \\
\hline Coahuila & 160911 & 21338 & 45092 & 1662 & 1580 & 82 & 5732 \\
\hline Colima & 55315 & 11617 & 24837 & 1036 & 1013 & 23 & 4110 \\
\hline Chiapas & 299239 & 35376 & 109774 & 4720 & 4661 & 59 & 15620 \\
\hline Chihuahua & 163353 & 20729 & 43846 & 1718 & 1580 & 138 & 4350 \\
\hline Distrito Federal & 259693 & 88060 & 86974 & 11069 & 9955 & 1114 & 23507 \\
\hline Durango & 132862 & 16894 & 51684 & 1431 & 1358 & 73 & 4127 \\
\hline Guanajuato & 303808 & 40462 & 91342 & 4105 & 4018 & 87 & 10422 \\
\hline Guerrero & 560838 & 39538 & 171185 & 5333 & 5189 & 144 & 9510 \\
\hline Hidalgo & 221549 & 23660 & 80591 & 2760 & 2619 & 141 & 5668 \\
\hline Jalisco & 458080 & 57341 & 158160 & 5223 & 4869 & 354 & 9418 \\
\hline México & 1541750 & 92886 & 510699 & 7827 & 7455 & 372 & 27932 \\
\hline Michoacán & 320458 & 44810 & 102785 & 3877 & 3640 & 237 & 10455 \\
\hline Morelos & 138665 & 13029 & 51181 & 2101 & 2023 & 78 & 4836 \\
\hline $\mathrm{N}$ ayarit & 141802 & 16843 & 40896 & 1252 & 1187 & 65 & 2934 \\
\hline N uevo León & 210288 & 32185 & 80147 & 2862 & 2714 & 148 & 12403 \\
\hline 0 axaca & 283861 & 29142 & 102430 & 3104 & 2993 & 111 & 7423 \\
\hline Puebla & 500975 & 42854 & 145467 & 4581 & 4284 & 297 & 8549 \\
\hline Q uerétaro & 80523 & 11023 & 39391 & 1918 & 1816 & 102 & 3488 \\
\hline Q uintana Roo & 88251 & 10440 & 24670 & 1440 & 1377 & 63 & 3053 \\
\hline San Luis Potosí & 152132 & 20583 & 50706 & 2061 & 1967 & 94 & 6288 \\
\hline Sinaloa & 129835 & 20219 & 49160 & 2178 & 2100 & 78 & 3796 \\
\hline Sonora & 130673 & 17252 & 45787 & 2363 & 2227 & 136 & 5109 \\
\hline Tabasco & 278002 & 23007 & 130647 & 4730 & 4557 & 173 & 9176 \\
\hline Tamaulipas & 160149 & 21132 & 43733 & 4182 & 4026 & 156 & 21327 \\
\hline Tlaxcala & 106235 & 10758 & 50097 & 2153 & 2086 & 67 & 4885 \\
\hline Veracruz & 548800 & 73229 & 239110 & 10099 & 9991 & 108 & 27323 \\
\hline Yucatán & 105536 & 10954 & 42557 & 1651 & 1583 & 68 & 3939 \\
\hline Zacatecas & 147105 & 16392 & 51183 & 1227 & 1159 & 68 & 4093 \\
\hline Institutos nacionales & 5253 & 0 & 0 & 1294 & 1203 & 91 & 3181 \\
\hline
\end{tabular}




\section{Accidentes atendidos. México, 2000}

\begin{tabular}{|c|c|c|c|c|c|c|c|}
\hline Entidad federativa & Total & $<5$ años & De 5 a 9 años & De 10 a 19 años & De 20 a 49 años & De 50 a 64 años & De 65 y más \\
\hline Estados Unidos Mexicanos & 879191 & 107992 & 120363 & 215682 & 326293 & 64787 & 44074 \\
\hline A guascalientes & 19761 & 2684 & 2806 & 5226 & 6669 & 1295 & 1081 \\
\hline Baja California & 12659 & 1312 & 1323 & 2721 & 5903 & 893 & 507 \\
\hline Baja C alifornia Sur & 5165 & 575 & 573 & 1169 & 2412 & 290 & 146 \\
\hline Campeche & 8499 & 664 & 1033 & 2076 & 3902 & 518 & 306 \\
\hline Coahuila & 13603 & 2898 & 1963 & 2933 & 4413 & 799 & 597 \\
\hline$\overline{C o l i m a}$ & 5227 & 574 & 663 & 1335 & 2007 & 377 & 271 \\
\hline Chiapas & 22127 & 1821 & 2537 & 5819 & 9579 & 1538 & 833 \\
\hline Chihuahua & 26680 & 2981 & 3439 & 6036 & 11208 & 1860 & 1156 \\
\hline Distrito Federal & 121435 & 17882 & 14111 & 26831 & 49180 & 8429 & 5002 \\
\hline Durango & 10686 & 1273 & 1626 & 2887 & 3593 & 726 & 581 \\
\hline Guanajuato & 52924 & 6151 & 7386 & 14458 & 18123 & 3815 & 2991 \\
\hline Guerrero & 43865 & 4157 & 5865 & 11603 & 15822 & 3827 & 2591 \\
\hline Hidalgo & 35951 & 3299 & 5162 & 8664 & 12960 & 3542 & 2324 \\
\hline Jalisco & 27158 & 3370 & 3927 & 7103 & 9226 & 1807 & 1725 \\
\hline México & 95826 & 12530 & 14647 & 23927 & 33909 & 6479 & 4334 \\
\hline Michoacán & 39277 & 4067 & 5251 & 10057 & 14643 & 2911 & 2348 \\
\hline Morelos & 32303 & 4942 & 4970 & 7822 & 10862 & 2065 & 1642 \\
\hline $\mathrm{N}$ ayarit & 12219 & 1387 & 1653 & 3112 & 4442 & 924 & 701 \\
\hline Nuevo León & 15093 & 2572 & 2662 & 3361 & 4816 & 997 & 685 \\
\hline 0 axaca & 37575 & 3072 & 4976 & 9627 & 13987 & 3631 & 2282 \\
\hline Puebla & 25693 & 2231 & 3464 & 6818 & 9455 & 2228 & 1497 \\
\hline Q uerétaro & 17057 & 2030 & 2442 & 4254 & 6221 & 1214 & 896 \\
\hline Q uintana Roo & 11548 & 1293 & 1422 & 2843 & 4863 & 756 & 371 \\
\hline San Luis Potosí & 16533 & 1728 & 2561 & 3963 & 5588 & 1633 & 1060 \\
\hline Sinaloa & 14111 & 2009 & 2330 & 3267 & 4659 & 1032 & 814 \\
\hline Sonora & 28173 & 4432 & 4253 & 6873 & 10146 & 1589 & 880 \\
\hline Tabasco & 22954 & 2046 & 2775 & 5756 & 10089 & 1474 & 814 \\
\hline Tamaulipas & 24383 & 4787 & 4066 & 5651 & 7427 & 1495 & 957 \\
\hline Tlaxcala & 9856 & 963 & 1288 & 2543 & 3940 & 641 & 481 \\
\hline Veracruz & 46663 & 5775 & 6079 & 10986 & 17334 & 3988 & 2501 \\
\hline Yucatán & 15853 & 1424 & 1935 & 3881 & 6128 & 1318 & 1167 \\
\hline Zacatecas & 8334 & 1063 & 1175 & 2080 & 2787 & 696 & 533 \\
\hline
\end{tabular}

Fuente: Secretaría de Salud (SSA), C oordinación de Planeación Estratégica, D irección General de Información y Evaluación del D esempeño (DGIE), Sistema de Información en Salud para Población A bierta (SISPA) 


\section{Detección de enfermedades. México, 2000}

Entidad federativa

\begin{tabular}{|c|c|c|c|c|c|c|c|}
\hline \multirow{2}{*}{$\begin{array}{l}\text { Cáncer } \\
\text { cervicouterino }\end{array}$} & \multirow{2}{*}{$\begin{array}{l}\text { Cáncer } \\
\text { de mama }\end{array}$} & \multicolumn{2}{|c|}{ Diabetes mellitus } & \multicolumn{2}{|c|}{ Hipertensión arterial } & \multicolumn{2}{|c|}{ Tuberculosis } \\
\hline & & Hombres & M ujeres & Hombres & M ujeres & Hombres & M ujeres \\
\hline 2323336 & 3053912 & 1413766 & 3357115 & 2290303 & 5871730 & 189725 & 237986 \\
\hline
\end{tabular}

Estados Unidos Mexicanos

21261

22948

$10261 \quad 26094$

$16988 \quad 47741$

$646 \quad 920$

Baja California

49068

Baja California Sur

14231

42932

20228

52209

10081

19653

33836

95376

$12016 \quad 25003$

$33806 \quad 11849$

25561

12645

30573

Coahuila

Chiapas

40682

47003

28026

61761

27548

67514

$21513 \quad 18625 \quad 45663$

$14850 \quad 35556$

$83584 \quad 112990$

Chihuahua

44849

53787

52049

103298

$111697 \quad 254665$

Distrito Federal

189080

204578

35939

74913

44845

254665

Durango

3499

51917

84802

229551

148528

102703

$33008 \quad 91849$

$21371 \quad 44868$

201972

\begin{tabular}{ll}
\hline Guanajuato & 125607 \\
\hline Guerrero & 139495
\end{tabular}

Hidalgo 98688

Jalisco

131971

México

151198

Michoacán

87556

Morelos

39761

$\mathrm{N}$ ayarit

53784

151453

65264

191500

$88614 \quad 284293$

$\begin{array}{llll}88614 & 284293 & 4691 & 6720\end{array}$

Nuevo León

\begin{tabular}{ll}
\hline axaca & 80 \\
\hline Puebla & 1132
\end{tabular}

Querétaro

87811

$80078 \quad 118086$

$113288 \quad 165638$

44128

42812

44128

84490

146237

$87916 \quad 204044$

$10327 \quad 14626$

Q uintana Roo

33459

39431

68035

120762

$148811 \quad 337176$

$7717 \quad 12128$

San Luis Potosí

56556

Sinaloa

57798

Sonora

50437

68516

68569

$134119 \quad 347369$

$76814 \quad 219969$

$\begin{array}{rr}7717 & 12128 \\ 11293 & 13762\end{array}$

Tabasco

105236

53407

109016

$60117 \quad 8336$

Tlaxcala

Veracruz

44353

65883

$154313 \quad 230510$

31553

33699

Zacatecas

46099

52944

Fuente: Secretaría de Salud (SSA), C oordinación de Planeación Estratégica, D irección General de Información y Evaluación del Desempeño (D G IE), Sistema de Información en Salud para Población A bierta (SISPA) 